\title{
Enhancement of afferent fiber activity in hippocampal slices
}

\author{
WALTER C. LOW* and SPENCER L. BEMENT \\ Bioelectrical Sciences Laboratory, University of Michigan, Ann Arbor, Mich. 48109 (U.S.A.)
}

(Accepted June 12th, 1980)

Key words: hippocampus — potentiation - radiatum fibers

The potentiation of synaptic activity in the hippocampal formation is a well documented phenomenon. It has been suggested, however, that a recruitment of additional afferent fibers can contribute to such an increase in synaptic activity. The hypothesis of an enhancement in afferent fiber activity, therefore, was investigated with the in vitro hippocampal slice preparation. Isolated radiatum fiber compound action potentials were evoked with paired electrical stimuli of equal intensity and separated in time by $30-40 \mathrm{msec}$. Statistical comparisons between control and test evoked responses reveal an augmentation of the test responses in support of the hypothesis of an enhancement in afferent fiber activity.

The potentiation of synaptic activity within the hippocampal formation has been revealed by the recording of extracellular postsynaptic field potentials ${ }^{1,3,11}$ and constitutes one type of neurophysiological plasticity. An enhancement in afferent fiber activity, however, may contribute to the potentiation of synaptic activity. In this study the synchronous compound action potential arising from the activation of fibers in stratum radiatum ${ }^{2}$ is used to test this hypothesis.

The compound action potential or afferent volley, however, is superimposed upon the summated extracellular excitatory postsynaptic potentials (EPSPs) when recorded in stratum radiatum. In order to investigate the possible enhancement of afferent activity, a measure of the afferent volley is required that is free of distortions arising from the summated EPSPs.

In this regard, a putative radiatum afferent volley was isolated and examined without the initiation of synaptic transmission and the resulting extracellular field potentials. The putative afferent volley was examined with respect to its waveform, spatial localization, conduction velocity, and 'absolute refractory period'2. It was concluded that the putative volley was generated by radiatum fiber activity. Analysis of the isolated radiatum afferent volley with a paired pulse paradigm suggested that the activity of the afferent fibers can be enhanced and therefore contribute to the enhancement of synaptic activity.

Hippocampal slices from Sprague-Dawley rats were prepared in a manner similar to that described by Yamamoto ${ }^{12}$. The hippocampus was sectioned transverse

* Present address: Department of Physiology and Biophysics, University of Vermont, Burlington, Vt. 05401 , U.S.A. 
to its septo-temporal axis and the slices placed in a non-aerated glucose-Ringer solution (124 mM NaCl, $5 \mathrm{mM} \mathrm{KCl}, 1.24 \mathrm{mM} \mathrm{KH}_{2} \mathrm{PO}_{4}, 1.3 \mathrm{mM} \mathrm{MgSO}_{4}, 2.4 \mathrm{mM}$ $\mathrm{CaCl}_{2}, 26 \mathrm{mM} \mathrm{NaHCO}$ and $10 \mathrm{mM}$ glucose) at room temperature for 5-10 min. This anoxic condition was found to arrest the process of synaptic transmission, but not the evoked radiatum fiber activity. The slices were then placed in the inner well of a warmed $\left(37 \pm 0.5^{\circ} \mathrm{C}\right)$ incubation chamber also containing the glucose-Ringer. A gaseous mixture of $95 \% \mathrm{O}_{2}$ and $5 \% \mathrm{CO}_{2}$ was bubbled into the outer well of the chamber containing distilled water.

Insulated bipolar stimulating electrodes (40-gauge nichrome) were visually placed in stratum radiatum of the subfield CAl. An isolated constant current source driven by a Grass stimulator (Model SD5) was used to excite the radiatum fibers. Current pulses of $100 \mu \mathrm{sec}$ duration and $10 \mu \mathrm{A}-5 \mathrm{~mA}$ in intensity were used to evoke responses from stratum radiatum. A glass capillary recording electrode (1-3 M $\Omega$ ) filled with $2 \mathrm{M} \mathrm{NaCl}$ was also placed in stratum radiatum of CA1 but more distal to subfield $\mathrm{CA} 3$ than the stimulating electrodes. The detected evoked responses were amplified via a high impedance pre-amplifier (Transidyne General 1600) and recorded on the FM channel of a HP3900 tape recorder for later processing. Paired stimuli separated by $30-40 \mathrm{msec}$ in time were applied once every $2 \mathrm{sec}$. The current pulses were monitored with an oscilloscope to ensure that the amplitude of the paired stimuli were identical and not altered by electrolysis.

Each recorded evoked response was digitized and stored on a computer disk file with a HP $21 \mathrm{MX}$ computer system. The digitized responses were analyzed to determine the evoked amplitudes using algorithms to measure the peak negativity of the radiatum response. The measured amplitudes for test and control responses were analyzed statistically with computer based programs.

The characteristic triphasic waveform of an isolated radiatum afferent volley is illustrated in Fig. 1A. The latency of the afferent volley from the stimulus artifact to the peak negativity was approximately $1.0-1.5 \mathrm{msec}$ for distances of $200-250 \mu \mathrm{m}$ between the stimulating and recording electrodes. Afferent volley amplitudes were usually quite small, and ranged from 100 to $700 \mu \mathrm{V}$, with a duration of approximately $1 \mathrm{msec}$. The form of the afferent volley when the polarity of the stimulus was inverted was similar to the non-inverting stimulus response, suggesting that it was not a stimulus artifact. The radiatum afferent volley identified by Andersen and his associates $^{2}$ in conjunction with the population EPSP is illustrated in Fig. 1B. Note the similarity in waveforms and time course of the radiatum and putative afferent volley as shown in traces $\mathrm{B}$ and $\mathrm{A}$ respectively.

The 'absolute refractory period' of the putative afferent volley was obtained by reducing the time interval between paired stimuli of identical intensity and duration until the volley disappeared. The 'absolute refractory period' was found to range from 2 to $3 \mathrm{msec}$, as illustrated in the traces of Fig. 1C.

When the recording electrode was incremented across the surface of the slice from stratum pyranidale to the hippocampal fissure, the negative phase of the putative afferent volley varied in size as illustrated in the graph of Fig. 1D. The region exhibiting maximal negativity reflects the location of the active current sink and was visually localized to stratum radiatum. 
A

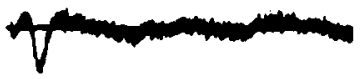

B

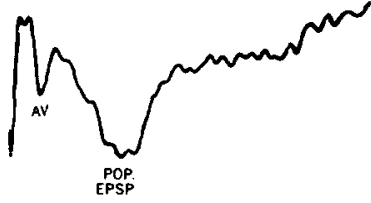

C

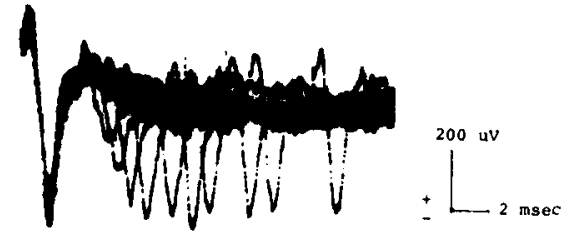

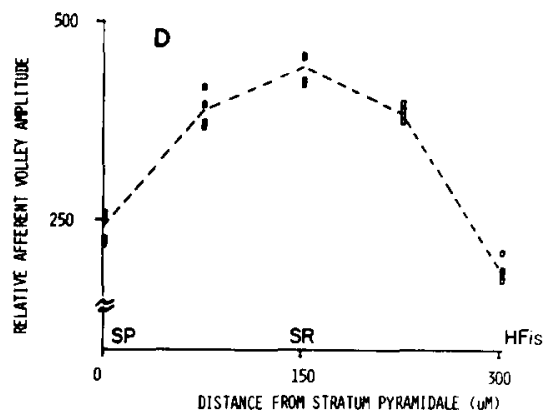

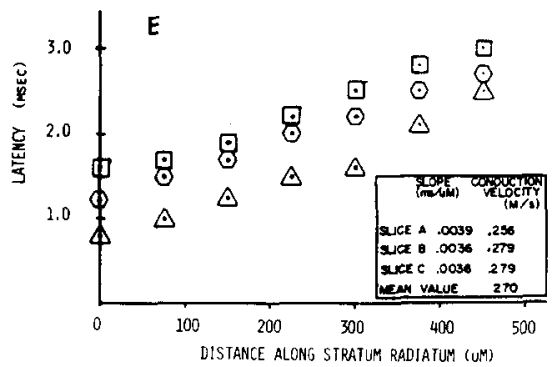

Fig. 1. Waveform characteristics, spatial localization and latency measurements of afferent volleys. A: putative volley recorded from stratum radiatum. B: radiatum volley (AV) and population EPSP. C: "absolute refractory period" of the putative afferent volley as determined from afferent volley responses to paired stimuli with decreasing time intervals between the stimulus pair. The disappearance of the test response occurs at a $2-3$ msec interval between the paired stimuli. D: plot of the putative afferent volley negativity as a function of distance from stratum pyramidale. Four responses are recorded at each spatial location. SP, stratum pyramidale; SR, stratum radiatum; HFis, hippocampal fissure. E: plot of the latency of the putative afferent volley as a function of distance along stratum radiatum. Measurements are relative to the initial recording position. Open squares, slice A; open hexagons, slice B; open triangles, slice $\mathrm{C}$.

When the recording electrode was incremented within stratum radiatum, parallel to the radiatum fibers and away from the stimulating electrode, latency increases in the afferent volley were observed. A plot of latency as a function of recording electrode position is illustrated in Fig. $1 \mathrm{E}$ for 3 different slices. The slopes determined from a least squares fit of the data are shown in the inset of Fig. 1E along with the associated conduction velocities. The mean conduction velocity was $0.270 \mathrm{~m} / \mathrm{sec}$ which is consistent with fiber tracts in this area ${ }^{2}$.

On the basis of the spatial localization studies, the evoked response seems to be restricted to within the region traversed by the radiatum fibers. Further, the waveform duration $(1 \mathrm{msec})$, and latency $(1 \mathrm{msec})$ of the putative afferent volley are also similar to those of the radiatum afferent volley determined by Andersen et al. ${ }^{2}$. Finally, the mean fiber conduction velocity of $0.270 \mathrm{~m} / \mathrm{sec}$ and 'absolute refractory period' of 2-3 msec are also comparable to the $0.3 \mathrm{~m} / \mathrm{sec}$ and the $2 \mathrm{msec}$ values determined by Andersen and his associates ${ }^{2}$. These observations suggest that the evoked response recorded in stratum radiatum, after procedures to abolish synaptic transmission, represent the synchronous compound action potential of the radiatum fibers. 

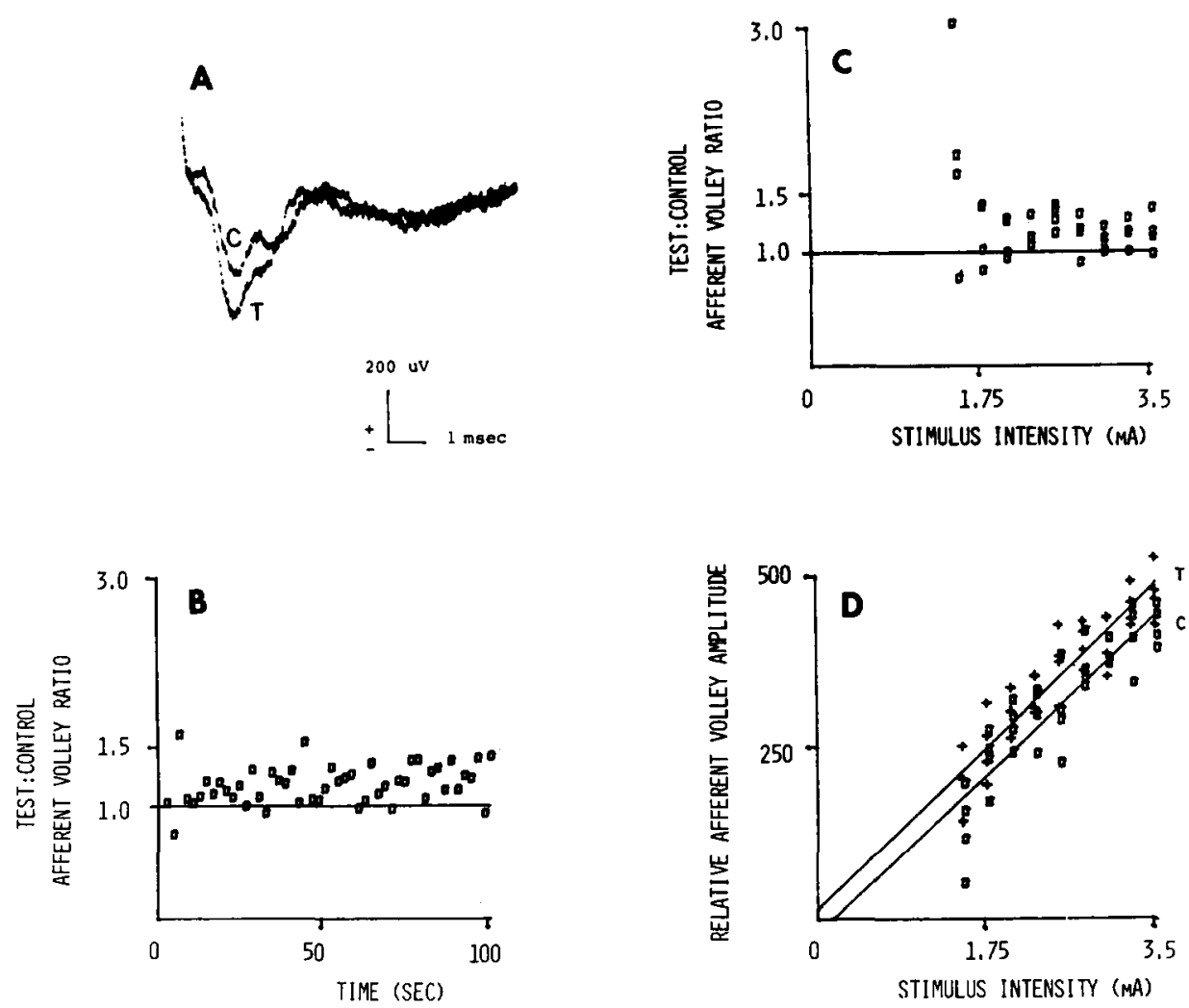

Fig. 2. Enhancement of afferent activity. A: control (C) and test (T) afferent volleys from paired stimuli of equal intensity, but separated in time by $40 \mathrm{msec}$. B: afferent volley test : control ratios over a $100 \mathrm{sec}$ time period. $\mathrm{C}$ : afferent volley test : control ratios with increasing stimulus intensities. $\mathrm{D}$ : afferent test (crosses) and control (open squares) responses with increasing stimulus intensities. Regression line $\mathrm{T}$, test responses; regression line $\mathrm{C}$, control responses.

The radiatum afferent volleys from paired stimuli separated in time by $30-40$ msec generally exhibited potentiation of the test response as shown in Fig. 2A. The ratio between the test and control afferent volley amplitudes was determined over a $100 \mathrm{sec}$ time period with constant intensity paired stimuli applied once every $2 \mathrm{sec}$. In general, the test: control ratios were found to be greater than 1.0, as illustrated in Fig. $2 \mathrm{~B}$. Of the response pairs $88 \%$ had ratios greater than 1.0 . Afferent volley potentiation was also supported by comparisons of mean test and control values over the $100 \mathrm{sec}$ time period for 15 slices; 14 of the 15 slices exhibited a mean test response greater than the mean control response. When the 15 slices are considered as a group the hypothesis that the mean value of the test responses is equal to or less than the mean value of the control responses $\left(\mathrm{H}_{0}: \mathrm{AVt}-\mathrm{AVc}=0\right)$ was tested with the Student $t$-statistic at $\alpha=$ 0.05 and rejected.

Paired stimuli of varying intensities were used to examine the potentiation of the afferent volley over a wide range of applied stimuli. The test:control ratio again indicated that paired responses were generally potentiated, as shown in Fig. $2 \mathrm{C}$ where 28 of 36 response pairs exhibited test: control ratios greater than 1.0 . 
Afferent volley potentiation was also supported by statistical comparison of regression lines fitted to the control and test data over a linear range of responses, as shown in Fig. 2D. The hypothesis of a similarity in slope between the test and control regression lines was tested with a modified $t$-statistic ${ }^{4}$. This hypothesis could not be rejected $(P<0.05)$ in each of the 15 slices and suggested that these responses were parallel. However, the hypothesis of a similarity in intercept between the parallel control and test regression lines (also tested with a modified $t$-statistic $\left.{ }^{4}\right)$ was rejected $(P$ $<0.05$ ) in 13 of the 15 cases indicating that although the control and test regression lines were parallel, they were not coincident. The 13 control and test regression line pairs with statistically different intercepts all exhibited a test intercept greater than the associated control intercept, which suggests a potentiation of the test responses.

The high degree of linearity and the consistent parallel nature between the control and test functions suggest that possibly a fixed proportion of the total number of afferents may be enhanced during the potentiation process. This may, in part, account for the decrease in the degree of enhancement at high stimulus intensities.

The enhancement of afferent fiber activity has not been previously observed by others studying the hippocampal formation. Lømo ${ }^{10}$, in his examination of the perforant path fibers of the dentate using an acute preparation, did not observe the recruitment of additional fibers with paired pulse stimulation. However, recording a synchronous compound action potential in the central nervous system is quite difficult because of the short distance required between the stimulating and recording electrodes and is therefore not amenable to acute recording techniques.

Andersen et al. $^{3}$, on the other hand, used in vitro techniques to examine the possibility of enhanced radiatum fiber activity following a tetanic stimulation paradigm. Post-tetanic enhancement of radiatum fiber activity was not detected. This observation may indicate that enhanced fiber activity is indeed a short lasting phenomenon.

The enhancement of fiber activity, however, has been observed in isolated frog nerves ${ }^{8}$, cat hypogastric nerves ${ }^{9}$, earth worm giant fibers ${ }^{5}$, and cerebeliar parallel fibers in cats ${ }^{7}$. Moreover, the time course of enhancement for these various fibers ranged from 10-30 msec for cerebellar parallel fibers to $25-60 \mathrm{msec}$ for cat hypogastric nerves and are comparable with the duration of enhanced radiatum fiber activity.

A further comparison between hippocampal radiatum and cerebellar parallel fiber characteristics reveals additional similarities. Both types are unmyelinated with diameters less than $1 \mu \mathrm{m}$, and exhibit conduction velocities of about $0.3 \mathrm{~m} / \mathrm{sec}$. Also, both give rise to dilated en passant synapses. The packing density of parallel fibers is $10-20$ fibers/sq. $\mu \mathrm{m}$, and a similar packing density is likely for radiatum fibers. With such packing densities, fiber activity may be sensitive to alterations in the extracellular environment, as suggested by Gardner-Medwin? ${ }^{7}$. Alterations in fiber excitability have been demonstrated in the squid axon with a reduction in extracellular calcium ${ }^{6}$. Specifically, a reduction in extracellular calcium was found to lower the threshold for the initiation of the impulse with a five-fold reduction in calcium depolarizing the membrane potential $10-15 \mathrm{mV}$. However, such a reduction in extracellular calcium within stratum radiatum during paired pulse activation has yet to be demonstrated. 
Regardless of the mechanism(s) involved, the enhancement of fiber activity is a phenomenon which can logically give rise to a potentiation of synaptic activity.

The invaluable discussions and programming asistance of Dr. David Whitehorn are gratefully acknowledged. This work was supported by a NIGMS predoctoral fellowship GM 01289 and a Rackham dissertation grant to W.C.L., and by USPHS Grant NS 08470.

1 Alger, B.E. and Teyler, T.J., Long-term and short-term plasticity in the CA1, CA3 and dentate regions of the rat hippocampal slice, Brain Research, 110 (1976) 463-480.

2 Andersen, P., Selvenius, H., Sundberg, S.H., Sveen, O. and Wigström, H., Functional characteristics of unmyelinated fibers in the hippocampal cortex, Brain Research, 144 (1978) 11-18.

3 Andersen, P., Sundberg, S.H., Sveen, O. and Wigström, H., Specific long-lasting potentiation of synaptic transmission in hippocampal slices, Nature, (Lond.), 266 (1977) 736-737.

4 Brownlee, K.A., Statistical Theory and Methodology in Science and Engineering, Wiley., New York, 1965, pp. 288-298.

5 Bullock, T.H., Facilitation of conduction rate in nerve fibers, J. Physiol. (Lond.), 114 (1951) 89-97.

6 Frankenhaeuser, B. and Hodgkin, A.L., The action of calcium on the electrical properties of squid axons, J. Physiol. (Lond.), 137 (1957) 218-244.

7 Gardner-Medwin, A.R., An extreme supernormal period in cerebellar parallel fibers, J. Physiol. (Lond.), 222 (1972) 357-371.

8 Graham, H.T., Supernormality, a modification of the recovery process in nerve, Amer.J. Physiol., 110 (1934) 225-242.

9 Grundfest, H. and Gasser, H.S., Properties of mammalian nerve fibers of slowest conduction, Amer J. Physiol., 123 (1938) 307-318.

10 Lømo, T., Potentiation of monosynaptic EPSPs in the perforant path granule cell synapse, Exp. Brain Res., 12 (1971) 46-63.

11 Steward, O., White, W.F., Cotman, C.W. and Lynch, G., Potentiation of excitatory synaptic transmission in normal and in the reinnervated dentate gyrus of the rat, Exp. Brain Res., 26 (1976) $423-441$.

12 Yamamoto, C., Activation of hippocampal neurons by mossy fiber stimulation in thin brain sections in vitro, Exp. Brain Res., 14 (1972) 423-435. 